\title{
Proceedings of the 5th Workshop on Automated Knowledge Base Construction (AKBC)
}

\section{Proceedings of the 5th Workshop on Automated Knowledge Base Construction (AKBC) at the 2016 Conference of the North American Chapter of the Association for Computational Linguistics: Human Language Technologies}

\section{Proceedings of the Workshop}


(C) 2016 The Association for Computational Linguistics

Order copies of this and other ACL proceedings from:

Association for Computational Linguistics (ACL)

209 N. Eighth Street

Stroudsburg, PA 18360

USA

Tel: $+1-570-476-8006$

Fax: +1-570-476-0860

acleaclweb.org

ISBN 978-1-941643-53-2 


\section{Introduction}

Extracting knowledge from Web pages and integrating it into a coherent knowledge base (KB) is a task that spans the areas of natural language processing, information extraction, information integration, databases, search, and machine learning. Recent years have seen significant advances in knowledge base construction, in both academia and industry. Many popular offerings, including digital assistants (Siri, Cortana, and Google Now) as well as search interfaces (Yahoo!, Bing, and Google) leverage semantic understanding and structured knowledge bases to respond to users. A similarly abundant set of knowledge systems have been developed at top universities such as Stanford (DeepDive), Carnegie Mellon (NELL), the University of Washington (OpenIE), the University of Mannheim (DBpedia), and the Max Planck Institut Informatik (YAGO, WebChild) among others. Our workshop serves as a forum for researchers on knowledge base construction in both academia and industry.

Unlike many other workshops, our workshop puts less emphasis on conventional paper submissions and presentations, but focuses on visionary papers and discussions, and structures the program around high-profile keynotes that foster discussion. In addition, one of the workshop's unique characteristics is its nomadic nature; AKBC has co-located with conferences which serve diverse communities, attesting to the broad appeal of the topic. Following the standalone AKBC 2010, AKBC 2012 (HLT-NAACL), AKBC 2013 (CIKM), and AKBC 2014 (NIPS), have each featured a dozen invited talks, drawn 2035 submissions, and attracted audiences of 75-100 from NLP, information extraction, and machine learning communities. Our speakers are similarly diverse, drawing from experts in knowledge base construction from academia, industry, and government agencies. AKBC has featured senior invited speakers from Google, Microsoft, Facebook, leading universities (MIT, Stanford, Univ. of Washington, CMU, Univ. of Massachusetts, and more), and DARPA. With this year's proposal, we would like to continue the tradition of bringing together researchers on the frontier of breakthrough research from different communities. By inviting established researchers for keynotes, and by focusing particularly on vision paper submissions, we aim to provide a vivid forum of discussion about the field of automated knowledge base construction.

Topics of Interest:

- machine learning on text; unsupervised, lightly- and distantly-supervised learning representation learning; distributional semantics; ontology construction

- human-computer collaboration in KB construction; automated population of wikis

- inference for graphical models and structured prediction; scalable approximate inference

- named entity extraction; relation extraction; (open) information extraction

- entity resolution; information integration; schema alignment; ontology alignment; monolingual alignment; alignment between KBs and text

- pattern analysis; semantic analysis of natural language; learning by reading

- databases; distributed information systems; probabilistic databases

- scalable computation; distributed computation

- queries on mixtures of structured and unstructured data; querying under uncertainty

- dynamic models; online adaptation of knowledge; temporal KBs; belief revision in KBs

- languages, toolkits and systems for automated KB construction;

- demonstrations of existing automatically-built KBs 



\section{Organizers:}

Jay Pujara, University of Maryland, College Park, USA

Tim Rocktaschel, University College London, UK

Danqi Chen, Stanford University, USA

Sameer Singh, University of Washington, USA

\section{Program Committee:}

Alan Akbik (Technical University of Berlin)

Gabor Angeli (Stanford University)

Stephen Bach (Stanford University)

Niranjan Balasubramanian (Stony Brook University)

Guillaume Bouchard (University College London)

Eunsol Choi (University of Washington)

Bhavana Dalvi (Allen Institute for Artificial Intelligence)

Doug Downey (Northwestern University)

Luis Galárraga (Télécom ParisTech University)

Matt Gardner (Carnegie Mellon University)

Annalisa Gentile (University of Sheffield)

Adam Grycner (Max-Planck-Institut für Informatik)

Roman Klinger (University of Stuttgart)

Sebastian Krause (German Research Centre for Artificial Intelligence)

Jayant Krishnamurthy (Carnegie Mellon University)

Omer Levy (Bar-Ilan University)

Victoria Lin (University of Washington)

Xiao Ling (University of Washington)

Daniel Lowd (Oregon State University)

Ndapa Nakashole (Carnegie Mellon University)

Jason Naradowsky (University College London)

Arvind Neelakantan (University of Massachusetts Amherst)

Hoifung Poon (Microsoft Research)

Pontus Stenetorp (University College London)

Partha Pratim Talukdar (Indian Institute of Science)

Niket Tandon (Max-Planck-Institut für Informatik)

Philippe Thomas (German Research Centre for Artificial Intelligence)

Larysa Visengeriyeva (Technische Universität Berlin)

Andreas Vlachos (University of Sheffield)

Bishan Yang (Carnegie Mellon University) 


\section{Invited Speakers:}

Antoine Bordes, Facebook Artificial Intelligence Research William Cohen, Carnegie Mellon University

Benjamin Van Durme, Johns Hopkins University

Oren Etzioni, Allen Institute for Artificial Intelligence

Percy Liang, Stanford University

Chris Manning, Stanford University

Andrew McCallum, University of Massachusetts Amherst

Kristina Toutanova, Microsoft Research 


\section{Table of Contents}

Using Graphs of Classifiers to Impose Constraints on Semi-supervised Relation Extraction

Lidong Bing, William Cohen, Bhuwan Dhingra and Richard Wang $\ldots \ldots \ldots \ldots \ldots \ldots \ldots . \ldots$

Discovering Entity Knowledge Bases on the Web

Andrew Chisholm, Will Radford and Ben Hachey..........................

IKE - An Interactive Tool for Knowledge Extraction

Bhavana Dalvi, Sumithra Bhakthavatsalam, Chris Clark, Peter Clark, Oren Etzioni, Anthony Fader

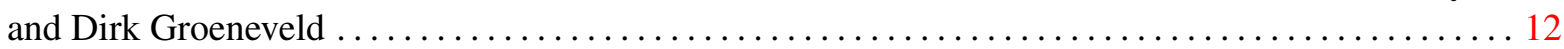

Incorporating Selectional Preferences in Multi-hop Relation Extraction

Rajarshi Das, Arvind Neelakantan, David Belanger and Andrew McCallum ............. 18

Knowledge Base Population for Organization Mentions in Email

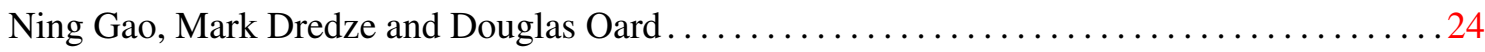

Enriching Wikidata with Frame Semantics

Hatem Mousselly Sergieh and Iryna Gurevych............................. 29

Demonyms and Compound Relational Nouns in Nominal Open IE

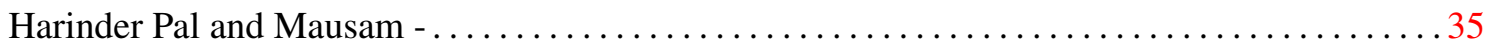

But What Do We Actually Know?

Simon Razniewski, Fabian Suchanek and Werner Nutt $\ldots \ldots \ldots \ldots \ldots \ldots \ldots \ldots \ldots \ldots \ldots$

Learning Knowledge Base Inference with Neural Theorem Provers

Tim Rocktäschel and Sebastian Riedel .................................. 45

The Physics of Text: Ontological Realism in Information Extraction

Stuart Russell, Ole Torp Lassen, Justin Uang and Wei Wang ...................... 51

Know2Look: Commonsense Knowledge for Visual Search

Sreyasi Nag Chowdhury, Niket Tandon and Gerhard Weikum .................... 57

Row-less Universal Schema

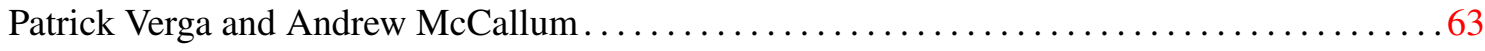

An Attentive Neural Architecture for Fine-grained Entity Type Classification

Sonse Shimaoka, Pontus Stenetorp, Kentaro Inui and Sebastian Riedel ................... 69

Regularizing Relation Representations by First-order Implications

Thomas Demeester, Tim Rocktäschel and Sebastian Riedel .................... 75

Applying Universal Schemas for Domain Specific Ontology Expansion

Paul Groth, Sujit Pal, Darin McBeath, Brad Allen and Ron Daniel ................. 81 
Design of Word Association Games using Dialog Systems for Acquisition of Word Association Knowledge

Yuichiro Machida, Daisuke Kawahara, Sadao Kurohashi and Manabu Sassano ............. 86

Call for Discussion: Building a New Standard Dataset for Relation Extraction Tasks

Teresa Martin, Fiete Botschen, Ajay Nagesh and Andrew McCallum..................92

A Comparison of Weak Supervision methods for Knowledge Base Construction

Ameet Soni, Dileep Viswanathan, Niranjan Pachaiyappan and Sriraam Natarajan .......... 97

A Factorization Machine Framework for Testing Bigram Embeddings in Knowledgebase Completion

Johannes Welbl, Guillaume Bouchard and Sebastian Riedel...................... 103 


\section{Conference Program}

Friday, June 17, 2016

9:00-9:10 Opening Remarks

AKBC Organizers

9:10-9:40 Joint Compositional Learning from Text and Knowledge Bases

Kristina Toutanova

9:40-10:10 The Allen AI Science Challenge: Results, Lessons, and Open Questions

Oren Etzioni

\section{0:10-11:00 Morning Poster Session and Coffee Break}

Using Graphs of Classifiers to Impose Constraints on Semi-supervised Relation Extraction Lidong Bing, William Cohen, Bhuwan Dhingra and Richard Wang

Discovering Entity Knowledge Bases on the Web

Andrew Chisholm, Will Radford and Ben Hachey

IKE - An Interactive Tool for Knowledge Extraction

Bhavana Dalvi, Sumithra Bhakthavatsalam, Chris Clark, Peter Clark, Oren Etzioni, Anthony Fader and Dirk Groeneveld

Incorporating Selectional Preferences in Multi-hop Relation Extraction

Rajarshi Das, Arvind Neelakantan, David Belanger and Andrew McCallum

Knowledge Base Population for Organization Mentions in Email

Ning Gao, Mark Dredze and Douglas Oard

Enriching Wikidata with Frame Semantics

Hatem Mousselly Sergieh and Iryna Gurevych

Demonyms and Compound Relational Nouns in Nominal Open IE

Harinder Pal and Mausam -

But What Do We Actually Know?

Simon Razniewski, Fabian Suchanek and Werner Nutt

Learning Knowledge Base Inference with Neural Theorem Provers

Tim Rocktäschel and Sebastian Riedel 
Friday, June 17, 2016 (continued)

11:00-11:30 Andrew McCallum's Mysterious Production of Facts (Talk TBA) Andrew McCallum

11:30-12:00 Look Ma, No Neurons: Using Explicit Inference Rules to Complete a KB William Cohen

\section{2:00-13:20 Lunch Break and Morning Posters}

\section{3:20-13:50 Contributed Talks 1}

13:20-13:35 The Physics of Text: Ontological Realism in Information Extraction Stuart Russell, Ole Torp Lassen, Justin Uang and Wei Wang

13:35-13:50 Know2Look: Commonsense Knowledge for Visual Search Sreyasi Nag Chowdhury, Niket Tandon and Gerhard Weikum

13:50-14:15 Meaningful Discourses (Talk TBA)

Christopher Manning

14:15-14:40 Common Sense and Language Benjamin Van Durme

\section{4:40-15:10 Contributed Talks 2}

14:40-14:55 Row-less Universal Schema

Patrick Verga and Andrew McCallum

14:55-15:10 An Attentive Neural Architecture for Fine-grained Entity Type Classification Sonse Shimaoka, Pontus Stenetorp, Kentaro Inui and Sebastian Riedel 
Friday, June 17, 2016 (continued)

\section{5:10-16:00 Afternoon Poster Session and Coffee Break}

Know2Look: Commonsense Knowledge for Visual Search

Sreyasi Nag Chowdhury, Niket Tandon and Gerhard Weikum

Regularizing Relation Representations by First-order Implications

Thomas Demeester, Tim Rocktäschel and Sebastian Riedel

Applying Universal Schemas for Domain Specific Ontology Expansion

Paul Groth, Sujit Pal, Darin McBeath, Brad Allen and Ron Daniel

Design of Word Association Games using Dialog Systems for Acquisition of Word Association Knowledge

Yuichiro Machida, Daisuke Kawahara, Sadao Kurohashi and Manabu Sassano

Call for Discussion: Building a New Standard Dataset for Relation Extraction Tasks Teresa Martin, Fiete Botschen, Ajay Nagesh and Andrew McCallum

The Physics of Text: Ontological Realism in Information Extraction

Stuart Russell, Ole Torp Lassen, Justin Uang and Wei Wang

An Attentive Neural Architecture for Fine-grained Entity Type Classification

Sonse Shimaoka, Pontus Stenetorp, Kentaro Inui and Sebastian Riedel

A Comparison of Weak Supervision methods for Knowledge Base Construction Ameet Soni, Dileep Viswanathan, Niranjan Pachaiyappan and Sriraam Natarajan

Row-less Universal Schema

Patrick Verga and Andrew McCallum

A Factorization Machine Framework for Testing Bigram Embeddings in Knowledgebase Completion

Johannes Welbl, Guillaume Bouchard and Sebastian Riedel 
Friday, June 17, 2016 (continued)

16:00-16:25 Querying Unnormalized and Incomplete Knowledge Bases

Percy Liang

16:25-16:50 Memory Networks for Language Understanding: Successes and Challenges Antoine Bordes

16:50-17:30 Afternoon Speaker Panel

Christopher Manning, Benjamin Van Durme, Percy Liang, Antoine Bordes

17:30-17:45 Closing Remarks

AKBC Organizers

\section{7:45-18:15 Evening Poster Session}

